\section{Vertebral Artery Dissection: Looking for the Ideal Study Protocol}

The article by Naggara et $\mathrm{al}^{1}$ on the added value of high-resolution MR imaging in the diagnosis of vertebral artery dissection shows an interesting comparison between routine clinical MR imaging examinations as performed in their institution and high-resolution imaging acquired with dedicated coils and different techniques. The study enhances the difficult diagnostic task of demonstrating the presence of a mural hematoma along the tortuous course of the vertebral artery together with the problem of differentiating it from a normal venous plexus.

The use of a multiparametric approach with different sequences together with dedicated coils increases the diagnostic accuracy in the article by Naggara et al. ${ }^{1}$ This article showed cases in which normal venous plexuses have been interpreted as mural hematomas with routine examinations and cases in which a vessel wall dissection was shown with the use of a high-resolution technique in vessels considered normal at routine examination.

In their study, the authors compared 2 different settings in which the same cases were studied first with contrast-enhanced MR angiography (CE-MRA) and T1-weighted imaging (T1WI) sequences with a regular coil and then with high-resolution T1WI, T2-weighted imaging (T2WI), proton attenuation-weighted imaging, and time-offlight (TOF) techniques with a superficial dedicated coil. As a consequence, the advantage of high signal intensity and high resolution obtained with dedicated coils is coupled with the advantage of performing added sequences.

The limitation of the CE-MRA acquisition in showing the vessel wall is known ${ }^{2}$ and is related to the use of short parameters that are required to perform first-pass studies; moreover, regarding the study of vertebral arteries, superimposition of venous structures and of venous plexuses can be present when the acquisition is not perfectly timed with the contrast bolus. The information we may obtain from CE-MRA is then similar to that obtained from digital subtraction angiography (ie, that of an indirect vessel stenosis determined by the invisible mural hematoma).

Fat-suppressed T1WI, as used by the authors of the study, may show the methemoglobin hematoma but may be disturbed by flowrelated enhancement as the authors state in the "Discussion" section, and the use of a presaturation band may be insufficient due to the very low, almost stagnant, flow in the venous plexuses.
The advantage of the high-resolution multiparametric approach performed with dedicated coils in the study relates to the possibility of comparing the different behaviors of mural hematomas using T1WI and T2WI and the added value of a 3D TOF sequence- - that is, sensitivity to high flow, presaturation of veins, and inflow-effect-based. In our experience, venous plexuses around vertebral arteries are never evident with this acquisition. Moreover because TOF techniques are T1WI, they are sensitive to the presence of methemoglobin and can show the mural hematoma if it is in the subacute phase.

An interesting aspect shown by the study is that venous plexuses at T1WI performed with dedicated coils are isointense with neck tissues, probably related to the use of a black-blood acquisition and/or gating association, not used in the fat-suppressed T1WI acquired routinely.

Again it seems that the use of more appropriate sequences, probably more than that of a dedicated coil, helps in the diagnosis of vertebral artery dissection.

Nevertheless, it is also true that the use of a high-resolution acquisition is important, especially for the study of vertebral arteries, frequently small in caliber.

The study of vertebral arteries is challenging, especially when dissection is suspected and appropriately designed examinations are needed. A multiparametric approach, with T1WI, T2WI, and TOF MRA followed by CE-MRA may be useful to identify the vessel pathology and differentiate hematomas and venous structures. Highresolution images with dedicated coils may be difficult to obtain in the acute phase, when most of the examinations are required.

\section{References}

1. Naggara O, Louillet F, Touzé E, et al. Added value of high-resolution MR imaging in the diagnosis of vertebral artery dissection. AJNR Am J Neuroradiol 2010;31:1707-12

2. Flis CM, Jager HR, Sidhu PS. Carotid and vertebral artery dissections: clinical aspects, imaging features and endovascular treatment. Eur Radiol 2007;17:820-34

N. Anzalone

Department of Neuroradiology Scientific Institute HS Raffaele Milan, Italy M. Cadioli Philips Medical System Best, the Netherlands

DOI 10.3174/ajnr.A2492 